\title{
Determination of palladium in tetrakis(triphenylphosphine) palladium catalyst samples using Teflon pressure vessel-assisted sample digestion and ICP-OES
}

\author{
Wei Wang ${ }^{1, a}$, Xiao Juan Wei ${ }^{2, b}$ \\ ${ }^{1}$ Zhejiang Province Key Laboratory of Nobel Metal Catalytic Materials and Technology, China \\ ${ }^{2}$ Zhejiang Metallurgical Research Institute, Hangzhou, Zhejiang, China \\ a570005029@qq.com, bjan1088@163.com
}

\begin{abstract}
Keywords: Palladium; Tetrakis (triphenylphosphine) palladium catalyst; Teflon pressure digestion vessel; ICP-OES
\end{abstract}

\begin{abstract}
A novel method for the determination of palladium in tetrakis (triphenylphosphine) palladium catalyst samples was established by inductively coupled plasma atomic emission spectrometry after samples digested by teflon pressure digestion vessel with aqua regia. Such experiment conditions were investigated as the influence of sample digestion methods, digestion time, digestion temperature and interfering ions on the determination. Under the optimized conditions, the linear range of calibration graph for Pd was $0 \sim 250.00 \mathrm{mg} \mathrm{L}-1$, and the recovery was $98.40 \%$ $103.60 \%$. The relative standard deviation (RSDs) for Pd was $1.78 \%$. The proposed method was applied to determine the same samples with atomic absorption spectrometry with the results consistently, which is suitable for the determination of palladium in tetrakis (triphenylphosphine) palladium catalyst samples.
\end{abstract}

\section{Introduction}

Platinum-group-metals (PGMs), especially Pt, Pd, and Rh, had been indispensable elements in various industrial catalyses. Tetrakis(triphenylphosphine) palladium catalysts with the characteristics of excellent hydrogenation performance were widely applied in petroleum chemical industry, fine chemical industry and organic synthesis. Palladium was the active center of the palladium catalyst, which influenced the catalytic performance of the catalyst. Quantitative determination of palladium in tetrakis(triphenylphosphine) palladium catalyst samples had a great significance to evaluate the catalytic performance of the catalyst. Hence, a reliable and efficient analytical technique was required for determining palladium in tetrakis(triphenylphosphine) palladium catalyst samples.

Several analytical techniques were applied for the determination of PGMs in various samples, such as cathodic stripping voltammetry (CSV) [1], neutron activation analysis (NAA) [2], atomic absorption spectrometry (AAS) [3-5], inductively coupled plasma optical emission spectrometry (ICP-OES) [6], inductively coupled plasma mass spectrometry (ICP-MS) [7, 8]. However, CSV was a powerful technique for the determination of ultratrace amounts of Pt, Pd and Rh, but the demands in terms of sample pretreatment were very stringent because the technique was very susceptible to interferences [3]. NAA could hardly be considered a routine approach due to the complexity and cost of the required instrumentation [4]. Electrothermal atomic absorption spectrometry (ETAAS) could also be applied for PGEs determinations in environmental and biological samples. However, such method suffers from severe matrix effects [5]. ICP-OES/MS was considered one of the most suitable instrumental techniques for determination of PGEs in different samples owing to its excellent analytical performance, such as multielement capability, wide linear dynamic range, low detection limit and good precision.

Another crucial step in the analysis of PGEs was sample digestion. The catalyst-derived particles, which carry PGEs, were usually in a form that was difficult to digest [7]. A number of papers had been published on the determination of PGEs directly from different environmental materials (road, dust, soil, plants and biological samples) [9-15]. The sample preparation methods used were typically 
based on microwave digestion with nitric acid, hydrofluoric acid and hydrochloric acid [16, 17]. However, a little of papers were published on the determination of PGEs from catalyst samples. T. M. Malyutina has developed a technique for the determination of platinum and palladium in dead platinum and palladium-loaded $\mathrm{Al}_{2} \mathrm{O}_{3}$ catalysts using ICP-OES after sample digestion by high temperature fusion with potassium pyrosulfate [18]. As far as we know, there was no report on the determination of palladium from tetrakis(triphenylphosphine) palladium catalyst samples, and using teflon pressure digestion vessel digestion with aqua regia in the literature. The aim of this work was to attempt to use aqua regia as digestion reagents to determine Pd in tetrakis(triphenylphosphine) palladium catalyst samples by ICP-OES with teflon pressure digestion vessel-assisted sample digestion, which could make organic compound oxided and palladium quantitative into the solution. The factors affecting experiment conditions were investigated as the influence of sample dissolution methods, digestion time, digestion temperature and interfering ions on the determination. The practical application of this method to the determination of Pd in tetrakis(triphenylphosphine) palladium catalyst samples was performed.

\section{Experimental}

Instrumentation. A Perkinelmer Optima 7000DV ICP-OES (Perkinelmer, America) was used in this work. The used instrument operating conditions and wavelength were given in Table 1 . The instrumental parameters were adjusted according to the manufacturer's recommendations.

In addition, an Analytik jena Nov AA 300 AAS (Analytikjena, Germany) was also used in the palladium determinations on the reference method. The instrumental parameters for AAS were as follows: wavelength Pd $247.6 \mathrm{~nm}$, slit $0.2 \mathrm{~nm}$, lamp current $8.0 \mathrm{~mA}$, Auxiliary gas $5.0 \mathrm{~L} \mathrm{~min}^{-1}$, Fuel gas $1.2 \mathrm{~L} \mathrm{~min}^{-1}$, EHT $433 \mathrm{~V}$, Burner height $6.0 \mathrm{~mm}$. The samples were digested in an MARS 6 microwave oven (CEM Corp., Matthews, NC, USA) equipped with easyprep plus extra high pressure Teflons TFM vessels (CEM Corp., $100 \mathrm{~mL}$, maximum pressure $15 \mathrm{MPa}$ and temperature $300{ }^{\circ} \mathrm{C}$ ) were used in the acid digestions of the samples. The microwave oven was operated in a temperature-controlled mode.

Table 1 ICP-OES operating conditions for determination of Pd

\begin{tabular}{|c|c|}
\hline Instrument parameter & \\
\hline Wavelength [nm] & 340.485 \\
\hline Incident power [kW] & 1.4 \\
\hline Cooling gas $\left[\mathrm{L} \min ^{-1}\right]$ & 15 \\
\hline Auxiliary gas $\left[\mathrm{L} \mathrm{min}^{-1}\right]$ & 0.2 \\
\hline Nebulizer gas $\left[\mathrm{L} \mathrm{min}^{-1}\right]$ & 0.8 \\
\hline Pump rate $\left[\mathrm{L} \mathrm{min}^{-1}\right]$ & 1.5 \\
\hline Nebulizer pressure $[\mathrm{kPa}]$ & 108 \\
\hline
\end{tabular}

Reagents and Sample Solutions. All reagents used were of analytical grade. Doubly distilled water was used throughout the experiment. Acidic standard $1000 \mathrm{mg} \mathrm{L}-1$ solutions of palladium (II) (Sigma-Aldrich, Germany) were used and working standard solutions were obtained by appropriate stepwise dilution of the stock standard solutions. The other reagents were as follows: HNO3 (65\%, Sinopharm), $\mathrm{HCl}$ (30\%, Sinopharm), Aqua regia was prepared by hydrochloric acid and nitric acid according to volume ratio 3:1.

Sample Digestion and Analytical Procedure. $0.10 \mathrm{~g}$ of tetrakis(triphenylphosphine) palladium sample was weighed into $100 \mathrm{~mL}$ teflon pressure digestion vessel and $20 \mathrm{~mL}$ aqua regia was added to the digestion vessels. The vessels were closed and the samples were digested in the drying oven for 8 hour at $180^{\circ} \mathrm{C}$. The solutions were carefully allowed to cool to room temperature prior to opening the vessels. The samples were heated to nearly dryness and then $10 \mathrm{~mL}$ of conc. $\mathrm{HCl}$ was added. The heating step was repeated three times. After that, the sample was diluted to $250 \mathrm{~mL}$ with volume ratio of $4 \% \mathrm{HCl}$ solution. 


\section{Results and Discussion}

Selection of Dissolution Methods. The same tetrakis(triphenylphosphine) palladium catalyst sample with the palladium content of $9.05 \%$ was pretreated by digestion method according to the recommended procedure and normal digestion method ( $0.10 \mathrm{~g}$ of tetrakis(triphenylphosphine) palladium catalyst sample was weighed into $100 \mathrm{~mL}$ beaker and $20 \mathrm{~mL}$ aqua regia was added to beaker, then the sample was digested for 1 hour on the electric hot plate. After digestion, the samples were heated to dryness and $10 \mathrm{~mL}$ of conc. $\mathrm{HCl}$ was added. The heating step was repeated three times. After that, the sample was diluted to $250 \mathrm{~mL}$ with volume ratio of $4 \% \mathrm{HCl}$ solution), and then determined by ICP-OES. The results were given in Table 2 . As could be seen, the result by Teflon pressure digestion vessel digestion method was in good agreement with the certified values, but normal digestion method cause the result on the low side. Because normal digestion method with low temperature and pressure could not completely digest palladium in tetrakis(triphenylphosphine) palladium catalyst sample. In this work, Teflon pressure digestion vessel digestion method was selected to pretreat samples.

Table 2 Comparison of the two dissolution methods

\begin{tabular}{cc}
\hline Method & Found [\%] \\
\hline Normal digestion method & 8.26 \\
Teflon pressure vessel digestion method & 9.04 \\
\hline
\end{tabular}

Effect of Digestion Time. Digestion time is an important parameter that influences the digestion of sample. A digestion time in the range of $2-10$ hour at $180{ }^{\circ} \mathrm{C}$ was investigated. A very short digestion time can not insure completely dissolution of sample. Therefore, 8 hour was chosen as optimum.

Effect of Digestion Temperature. Digestion temperature is also an important parameter that influences the digestion of sample. A digestion temperature in the range of $120-200{ }^{\circ} \mathrm{C}$ for 8 hour was investigated. A very low digestion temperature could not insure completely dissolution sample. Therefore, $180^{\circ} \mathrm{C}$ was chosen as optimum.

Interferences. The influence of $\mathrm{K}, \mathrm{Na}, \mathrm{Ni}, \mathrm{Co}, \mathrm{Al}, \mathrm{Fe}, \mathrm{Mg}$, $\mathrm{Ca}$ on the determination of $\mathrm{Pd}$ was investigated. The solutions of $1 \mathrm{mg} \mathrm{L}^{-1} \mathrm{Pd}(\mathrm{II})$ containing the corresponding other interfering ions were prepared and operated according to the recommended procedure. The results showed that the content of $\mathrm{K}, \mathrm{Na}, \mathrm{Ni}, \mathrm{Co}, \mathrm{Al}, \mathrm{Fe}, \mathrm{Mg}$, Ca up to 10 times more palladium had no significant effect on the determination of palladium. So the developed method was not affected by the potential interferences from interference elements.

Analytical Performance. With the use of established experimental parameters as shown in Table 1, the analytical performance of the method was evaluated. Experiment indicated palladium concentration within the range of $0 \sim 250 \mathrm{mg} / \mathrm{L}$ had a linear relation with emission intensity. The results of method recovery were given in Table 3 . The recovery was $98.40 \% \sim 103.60 \%$, and the average recovery was $100.30 \%$.

Table 3 Recoveries of samples with the addition of standard palladium

\begin{tabular}{ccccc}
\hline Sample No & Found $\left[\mathrm{mg} \mathrm{L}^{-1}\right]$ & Added $\left[\mathrm{mg} \mathrm{L}^{-1}\right]$ & Total Found $\left[\mathrm{mg} \mathrm{L}^{-1}\right]$ & Recovery [\%] \\
\hline 1 & 1.50 & 0.50 & 2.01 & 100.50 \\
2 & 2.00 & 0.50 & 2.59 & 99.67 \\
3 & 2.50 & 1.00 & 3.59 & 103.60 \\
4 & 3.50 & 1.50 & 4.92 & 98.40 \\
5 & 5.00 & 1.50 & 6.42 & 98.77 \\
6 & 7.50 & 2.00 & 9.58 & 100.84 \\
\hline
\end{tabular}


Sample Analysis. The practical tetrakis(triphenylphosphine) palladium catalyst sample was determined by ICP-OES and AAS, with the use of established experimental parameters. Repeat 6 times, the results were shown in table 4 . The results of two method was in good agreement with the certified values of $9.05 \%$, but the former is significantly higher than the latter in precision, which is an ideal kind of method for determination palladium in tetrakis(triphenylphosphine) palladium catalyst sample.

Table 4 Comparison of the two methods

\begin{tabular}{ccc}
\hline Method & Average Found [\%] & RSD [\%] \\
\hline ICP-OES & 9.03 & 1.78 \\
AAS & 8.99 & 2.98 \\
\hline
\end{tabular}

\section{Summary}

The method was established by using aqua regia as digestion reagents to determine $\mathrm{Pd}$ in tetrakis(triphenylphosphine) palladium catalyst samples by ICP-OES with teflon pressure digestion vessel-assisted sample digestion, which was simple, quick, high precision. A satisfactory result was obtained when applying it in the practical sample.

\section{Acknowledgements}

This work was financially supported by Zhejiang Province Key Laboratory of Noble Metal Catalytic Materials and Technology construction (2013F10008).

\section{References}

[1] R. Schierl: J. Microchem Vol. 67 (2000), p. 245

[2] X. Dai, C. Koeberl and H. Froschl: Anal. Chim. Acta Vol. 436 (2001), p. 79

[3] J. Ye, S. Liu, M. Tian, W. Li, B. Hu, W. Zhou and Q. Jia: Talanta Vol. 118 (2014), p. 231

[4] S.Z. Mohammadi, H. Hamidian and Z. Moeinadini: Cent. Eur. J. Chem Vol. 11 (2013), p. 1749

[5] J. Tilch, M. Schuster, M. Schwarzer and Fresenius’ J: Anal. Chem Vol. 367 (2000), p. 450

[6] P. Kovacheva and R. Djingova: Anal. Chim. Acta Vol. 464 (2002), p. 7

[7] I. Jarvis, M.M. Totland and K.E. Jarvis: Analyst Vol. 122 (1997), p. 19

[8] K. Kanitsar, G. Koellensperger, S. Hann, A. Limbeck, H. Puxbaum and G. Stingeder: J. Anal. Atom. Spectrom Vol. 18 (2003), p. 239

[9] S. Zimmermann, C.M. Menzel, Z. Berner, J.-D. Eckhardt, D. Stüben, F. Alt, J. Messerschmidt, H. Taraschewski and B. Sures: Anal. Chim. Acta Vol. 439 (2001), p. 203

[10] J.D. Whiteley and F. Murray: Sci. Total Environ Vol. 317 (2003), p. 121

[11]B. Sures, S. Zimmermann, J. Messerschmidt, A. Von Bohlen and F. Alt: Environ. Pollut Vol. 113 (2001), p. 341

[12] B. Sures, S. Zimmermann, C. Sonntag, D. Stuben and H. Taraschewski: Environ. Pollut Vol. 122 (2003), p. 401

[13]T. Meisel, N. Fellner and J. Moser: J. Anal. Atom. Spectrom Vol. 18 (2003), p. 720

[14]D. Cinti, M. Angelone, U. Masi and C. Cremisini: Sci. Total Environ Vol. 293 (2002), p. 47

[15] R. Djingova, H. Heidenreich, P. Kovacheva and B. Markert: Anal. Chim. Acta Vol. 489 (2003), p. 245

[16] K. Boch, M. Schuster, G. Risse and M. Schwarzer: Anal. Chim. Acta Vol. 459 (2002), p. 257

[17] O.V. Borisov, D.M. Coleman, K.A. Oudsema and R.O. Carter III: J. Anal. Atom. Spectrom Vol. 12 (1997), p. 239

[18] T.M. Malyutina, T.Y. Alekseeva, A.V. D’yachkova, G.S. Kudryavtseva, L.D. Berliner and Y.A. Karpov: Inorganic. Materials Vol. 46 (2010), p. 1479 
